\title{
BMJ Open Identification of the most vulnerable populations in the psychosocial sphere: a cross-sectional study conducted in Catalonia during the strict lockdown imposed against the COVID-19 pandemic
}

\author{
Judith Farrés (D) , ${ }^{1}$ Jose Luis Ruiz, ${ }^{1}$ Jose Manuel Mas, ${ }^{1}$ Lilibeth Arias, ${ }^{2,3}$ \\ Maria-Rosa Sarrias, ${ }^{4,5}$ Carolina Armengol, ${ }^{5,6}$ Pere-Joan Cardona, ${ }^{2,3,7}$ \\ Jose A Munoz-Moreno, ${ }^{8,9,10}$ Miriam Vilaplana, ${ }^{11}$ Belen Arranz, ${ }^{11,12,13}$ \\ Judith Usall, ${ }^{11,12}$ Antoni Serrano-Blanco, ${ }^{11,12,14}$ Cristina Vilaplana (D) ${ }^{2,3}$
}

To cite: Farrés J, Ruiz JL, Mas JM, et al. Identification of the most vulnerable populations in the psychosocial sphere: a cross-sectional study conducted in Catalonia during the strict lockdown imposed against the COVID-19 pandemic. BMJ Open 2021:11:e052140. doi:10.1136/ bmjopen-2021-052140

- Prepublication history and additional supplemental material for this paper are available online. To view these files, please visit the journal online (http://dx.doi.org/10.1136/ bmjopen-2021-052140).

Received 07 April 2021 Accepted 26 October 2021

Check for updates

(C) Author(s) (or their employer(s)) 2021. Re-use permitted under CC BY-NC. No commercial re-use. See rights and permissions. Published by BMJ.

For numbered affiliations see end of article.

Correspondence to Dr Cristina Vilaplana; cvilaplana@igtp.cat

\section{ABSTRACT}

Design and objectives A cross-sectional study to evaluate the impact of COVID-19 on the psychosocial sphere in both the general population and healthcare workers (HCWs).

Methods The study was conducted in Catalonia (Spain) during the first wave of the COVID-19 pandemic when strict lockdown was in force. The study population included all people aged over 16 years who consented to participate in the study and completed the survey, in this case a 74-question questionnaire shared via social media using snowball sampling. A total of 56656 completed survey questionnaires were obtained between 3 and 19 April 2020.

The primary and secondary outcome measures included descriptive statistics for the non-psychological questions and the psychological impact of the pandemic, such as depression, anxiety, stress and post-traumatic stress disorder question scores.

Results A n early and markedly negative impact on family finances, fear of working with COVID-19 patients and ethical issues related to COVID-19 care among HCWs was observed. A total of seven target groups at higher risk of impaired mental health and which may therefore benefit from an intervention were identified, namely women, subjects aged less than 42 years, people with a care burden, socioeconomically deprived groups, people with unskilled or unqualified jobs, patients with COVID-19 and HCWs working with patients with COVID-19.

Conclusions Active implementation of specific strategies to increase resilience and to prepare an adequate organisational response should be encouraged for the seven groups identified as high risk and susceptible to benefit from an intervention.

Trial registration number NCT04378452.

\section{INTRODUCTION}

By 30 March 2020, 78797 confirmed cases of SARS-CoV-2, 6528 deaths and 14709 patients who had recovered had been reported in

\section{Strengths and limitations of this study}

- The current study aimed to identify the impacts of the COVID-19 pandemic on a wide range of healthrelated dimensions 2 weeks after starting strict lockdown and while it was still in force.

- The survey rapidly reached a large number of people without exposing interviewers to infection, thus becoming one of the most extensive surveys ever published. A total of 56656 survey questionnaires were analysed, thus representing $0.85 \%$ of the Catalan population aged $>16$ years.

- The survey was long (74 questions), thus allowing to collect a large amount of data, but this might also have generated fatigue and a high drop-out rate.

- No validated scales were used.

- The snowball strategy via social media does not allow the study population to be controlled; therefore, this is not a representative survey of a specific population.

Spain. ${ }^{1}$ Of these, 16157 cases and 1410 deaths were recorded in Catalonia. ${ }^{2}$ The case fatality $(8 \%)$ was calculated using recorded cases, although the mortality rate was uncertain and the total number of cases was unknown. At that time, there was local transmission of SARS-CoV-2 in the community. Everyone with a compatible respiratory condition was considered likely to be a case of SARS-CoV-2, although an aetiological diagnosis was not possible for all suspected cases in the context of a health emergency because of the lack of diagnostic kits and saturation of the health system. $^{34}$

In this context, $16 \%$ of all cases confirmed in Catalonia by 30 March 2020 affected healthcare workers (HCWs). ${ }^{2}$ In addition to 
their obviously increased risk of being infected, frontline HCWs (emergency rooms, Intensive Care Units (ICUs) and other departments) fighting the SARS-CoV-2 epidemic were faced with high levels of stress and anxiety. This worsened as the tensions in the health systems increased, which required them to face important ethical dilemmas, including patient triage.

Previous major outbreaks of infectious diseases, such as Ebola, have demonstrated that they have an important impact at both an individual and a community level as health services, social systems and economic productivity are all severely affected. ${ }^{5}$ Indeed, an important impact on mental health and emotional burden as a result of the SARS-CoV-2 pandemic and mass quarantines, similar to those observed during other epidemics, has been reported. ${ }^{6-9}$ However, a certain degree of anxiety is necessary for the adoption of precautionary measures against infection outbreaks ${ }^{10}$ and to ensure the successful implementation of public health interventions. Additionally, the SARS epidemic showed that frontline HCWs suffered from chronic stress at the time and that this lasted for at least 1 year after the epidemic wave had receded. ${ }^{11}$

At the time of the strict lockdown in Spain, members of society and HCWs raised their concerns about how the outbreak and the measures implemented by the government were impacting people's lives. With the aim of assessing the nature of this effect and the hypothesis that it may be important in several health dimensions, we designed the present study in order to evaluate the impact of COVID-19 on the psychosocial sphere for both the general population and HCWs.

\section{MATERIALS AND METHODS Design and setting}

This is a cross-sectional study, conducted in Catalonia (Spain) in April 2020, during the first wave of the COVID-19 outbreak, 2 weeks after the implementation of strict lockdown and while this was still in force.

\section{Participants}

Anyone aged over 16 years willing to participate in the study and who gave consent by starting the questionnaire.

\section{Ethics}

Before starting the survey, participants were informed about the aim of the study, the compliance with their rights and the existence of Institutional Review Board (IRB) approval. They were also informed about their right of access, rectification, limitation and erasure of their personal data and to withdraw consent, as well as how to exercise any of these rights.

\section{Outcome measures}

Descriptive statistics for the non-psychological questions and depression, anxiety, stress and post-traumatic stress disorder (PTSD) scores to determine the psychological impact of the outbreak. The anonymous questionnaire was developed by the research team and included 74 questions (online supplemental table 1). To obtain demographical, health status and mental health data, questions reported in the literature were used. In contrast, questions to evaluate the socioeconomic sphere and habits during lockdown were created by the research team. A pilot test was conducted in order to evaluate the validity and reliability of the instrument and to detect any errors in its administration. The questionnaire was adjusted in light of these results before launch. The questionnaire was created using the Typeform software (Typeform SL, Barcelona, Spain) and complied with the European General Data Protection Regulation. The survey was shared in five different languages (Catalan, Spanish, English, Italian and French) via social media (WhatsApp, Telegram channels and institutional websites) using snowball sampling. HCW WhatsApp groups and telegram channels, as well as hospital institutional websites, were used to reach HCWs.

Completion of the whole questionnaire took approximately $10 \mathrm{~min}$. Initially, we estimated that approximately 2000 completed questionnaires within a period of 6 months (April-September 2020) would allow us to extract valid results. As we received a high number of completed questionnaires in just a few weeks, we analysed all completed questionnaires obtained between 3 and 19 April 2020. After collection, data were downloaded as a spreadsheet file (Excel Microsoft Office) and deleted from the Typeform software.

\section{Analysis and statistics}

All data were processed anonymously. Questionnaires in which the participant did not reach the end were considered to be incomplete and were discarded. Only finished questionnaires were saved and taken into account for the analysis. Individuals reaching the end of the questionnaire could leave questions unanswered. For individual questions, only the answers for that variable were considered. Questions were grouped into indices (socioeconomic precariousness index, depression index, anxiety index, stress index or PTSD) following the calculation detailed in online supplemental table S1). When computing a combined score for several questions, this score was only computed if all answers for it were present.

Since there were no specific criteria for age stratification or the population density that was significant for all questions, it was decided to divide these categories into groups with a similar sample size, thus resulting in the following age groups: <42, 42-52, 52-61 and >61 years. Given the volume of responses obtained, age ranges were determined statistically to ensure that they were homogeneous in terms of number of surveys completed per group. The scores for the socioeconomic precariousness index and population density (inhabitants $/ \mathrm{km}^{2}$ ) of the municipality where the respondents lived, as stated by the respondents, were also segmented into four groups each following the same strategy. The four score ranges established for the 0-19 socioeconomic precariousness scale 
were: low $\leq 7$ points, mid-low $=7-8.5$, mid-high $=8.5-10$ and high $>10$ points.

All results were obtained considering that the respondents were part of the totality of the cohort of respondents. Responses were also analysed by category and broken down into percentages according to conditional distributions, taking into account the gender of the respondents and their age group. We took the non-binary gender and those who preferred not to say which gender they identify as into account when analysing the results, as this enriches the conclusions. However, statistical analysis often does not take into account the minimum volumes of responses; therefore, only the groups of women and men were compared.

Response percentages were calculated based on the number of respondents for each answer out of the total number of responses to each question. To assess whether the categorical variables were significantly related or not, we applied the $\chi^{2}$ test independently to the counts observed. We conducted a bivariate analysis between scores and sociodemographic variables. Differences in score distribution between different groups were assessed by comparing probability distributions using a two-band Wilcoxon signed-rank test and calculating the $\mathrm{p}$ value using Matlab's 'signrank' function. ${ }^{12} 13$

All tests were applied bilaterally using a significance of $5 \%(\mathrm{p}<0.05)$.

\section{RESULTS}

\section{Characteristics of the cohort}

We analysed 56656 questionnaires. The characteristics of the cohort are described in table 1. Differences between categories by gender and age are presented in online supplemental table 2$)$. The majority of respondents were female $(70.4 \%)$ and from Catalonia $(95.63 \%$, with $27.7 \%$ being from Barcelona city), which represents $0.85 \%$ of the Catalan population aged $>16$ years. ${ }^{214}$

Those living most precariously were aged under 42 years, with $18.43 \%$ sharing an apartment/house $(p<0.01)$. Most respondents had a degree $(42.62 \%)$ and a qualified job $(36.13 \%)$. Around $9 \%$ of all respondents worked in the healthcare sector. Most unemployed people were in the younger age range $(7.6 \%)$ and in the non-binary/ those who preferred not to say groups (approximately $12 \%$ each).

Around $60 \%$ of all respondents declared that they were taking care of someone: $24.81 \%$ caring for children aged $<16$ years and $15.11 \%$ caring for parents. Women were caregivers more frequently than men $(\mathrm{p}<0.01)$. The burden of care was also higher for women and people aged $42-61$ years $(p<0.01)$ and worryingly high for $4.79 \%$ of all respondents.

\section{Impact of the pandemic on the general population}

The impact on the general population is described in tables 2 and 3 and online supplemental table 2). Thus, $85.32 \%$ of the cohort declared they were remaining at home. Those working in essential services were mostly women or of non-binary gender, and the percentage of women was also higher among those who were obliged to go to work on-site $(\mathrm{p}<0.01)$.

Only 2 weeks after starting the lockdown, $25 \%$ of the cohort had already lost their job. People aged less than 52 years, as opposed to those aged over 52 years, and men, as opposed to women, were the most affected $(\mathrm{p}<0.01)$. In addition, $20.67 \%$ of respondents declared that they had no savings at all (table 1). After the implementation of measures announced by the authorities to cope with the pandemic, $82.75 \%$ of respondents declared that they were being careful or had decreased their expenses. Up to $8.78 \%$ of respondents declared that they had used social services or that they would need to use them soon, with those aged less than 52 years and people identifying as non-binary or preferring not to say being the most affected. Respondents aged less than 42 years, followed by people aged over 61 years and people identifying as non-binary gender, had the highest precariousness index values $(\mathrm{p}<0.01)$.

Around $19.84 \%$ of respondents declared that they had come into contact with someone infected by SARS-CoV-2, half of them with a confirmed or probable case (more frequent for women aged less than 52 years, $\mathrm{p}<0.01)$. Similarly, $35.75 \%$ declared that they had used at least one existing healthcare resource or one put in place by the authorities in the context of the pandemic during the previous 14 days, and $73.82 \%$ reported having had one or more symptoms compatible with COVID-19. Less than $2 \%$ of people claiming to have had symptoms had undergone a PCR test. A greater percentage of women and those aged less than 42 years said that they felt worse at the moment they answered the survey compared with people in other groups $(\mathrm{p}<0.01)$.

Some $42.05 \%$ of respondents said they had increased their consumption habits, in most cases of food. Women aged less than 42 years showed the largest increase in consumption (except for illegal drugs) compared with other groups $(\mathrm{p}<0.01)$.

$\mathrm{TV}$, followed by social media, was the main source of information regarding the pandemic, with no significant differences being found between different genders or age groups. Around $26.82 \%$ of respondents declared that the information given did not accurately reflect reality (more frequent in women and people aged over 52 years $(\mathrm{p}<0.01)$, and a further $20.92 \%$ said that it was too negative or too sensationalist (more frequent in men and people aged less than 42 years $(\mathrm{p}<0.01)$. Similarly, $73.13 \%$ declared that they were afraid or worried, with this group including more women but a lower percentage of people aged over 61 years $(\mathrm{p}<0.01)$. Finally, $78.56 \%$ of the cohort declared that the pandemic had changed them, most of them $(50.41 \%)$ as regards the way that they see society/how we used to live. Those most affected were women (more than men) and those aged less than 42 years vs their counterparts aged $>61$ years $(p<0.01$ in both cases). 
Table 1 Characteristics of the cohort

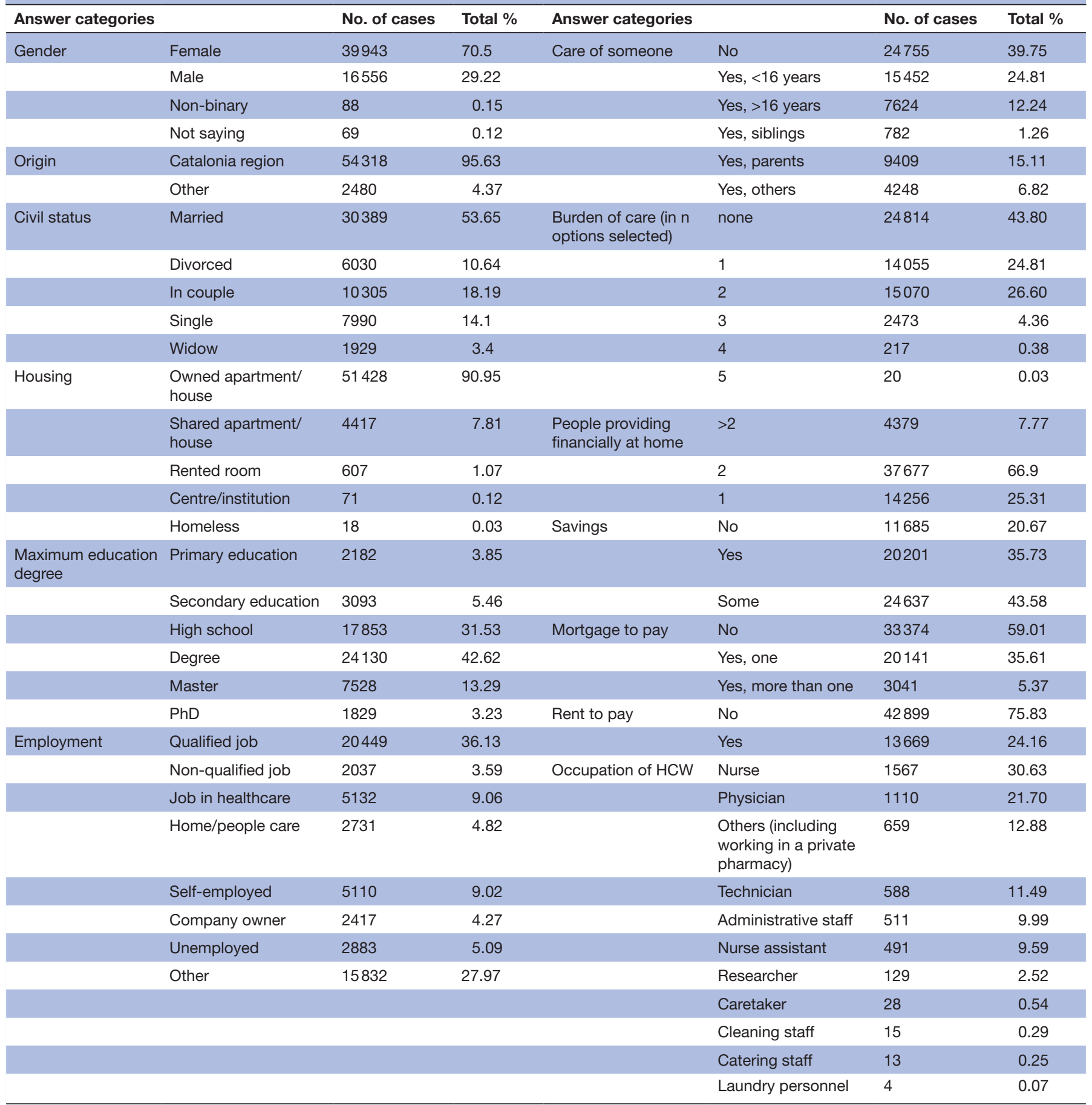

Number of cases (number of responses received per answer category) and percentage of the total responses obtained for each question. Please note that some of the questions were multiple choice.

$\mathrm{HCW}$, healthcare worker.

\section{Impact of the pandemic on HCWs}

A total of 5104 people $(9.05 \%$ of the total) identified themselves as workers in the healthcare sector, most of them being women. While the proportion women/men in the total cohort was $70 / 30$, in this subgroup the proportion was $85 / 15$. The impact on this population is detailed in table 4 . Thus, $41.65 \%$ of HCWs declared that they had worked directly with COVID-19 patients, 32\% of them while on duty. The majority of HCWs said that they were afraid to work with COVID-19 patients (75.87\%): $42.90 \%$ due to the risk of transmitting the infection to their relatives/friends, $17.07 \%$ due to the risk of getting infected or transmitting it to other patients and $4.28 \%$ due to the risk of dying. Surprisingly, fear of dying decreased with age. In all cases, higher percentages of younger HCWs said they were afraid $(\mathrm{p}<0.01)$. 
Table 2 Impact of the pandemic on the general population

\begin{tabular}{|c|c|c|c|}
\hline \multicolumn{2}{|l|}{ Answer categories } & \multirow{2}{*}{$\begin{array}{l}\text { No. of cases } \\
42475\end{array}$} & \multirow{2}{*}{$\begin{array}{l}\text { Total \% } \\
75.12\end{array}$} \\
\hline Loss of job & No & & \\
\hline & Yes, the company made a labour force adjustment plan & 103 & 0.18 \\
\hline & Yes, the company made a temporary labour force adjustment plan & 5530 & 9.78 \\
\hline & Yes, I have lost some previously contracted/arranged jobs & 3252 & 5.75 \\
\hline & Yes, I was fired & 499 & 0.88 \\
\hline & Yes, others & 4687 & 8.29 \\
\hline \multirow[t]{3}{*}{ Spending less } & Yes & 34307 & 60.66 \\
\hline & A little & 12493 & 22.09 \\
\hline & No & 9747 & 17.23 \\
\hline \multirow[t]{3}{*}{ Sought social assistance/or any other assistance } & No & 51588 & 91.00 \\
\hline & Not yet, but will need to & 2756 & 5.00 \\
\hline & Yes & 2208 & 4.00 \\
\hline \multirow{3}{*}{ Contact with someone infected by SARS-CoV-2 } & I do not know & 45,86 & 80.15 \\
\hline & Yes, with a probable non-confirmed case & 5627 & 9.83 \\
\hline & Yes, with a confirmed case & 5730 & 10.01 \\
\hline \multirow[t]{15}{*}{ Presence of symptoms (since February) } & No & 26598 & 26.18 \\
\hline & Headache & 16268 & 16.01 \\
\hline & Sore throat & 10013 & 9.85 \\
\hline & Nasal congestion/runny nose & 9322 & 9.17 \\
\hline & Extreme fatigue/tiredness & 7029 & 6.91 \\
\hline & Persistent cough (for 1 week or more) & 6957 & 6.84 \\
\hline & Muscle pain & 6299 & 6.20 \\
\hline & Diarrhoea & 5453 & 5.36 \\
\hline & Dizziness & 2897 & 2.85 \\
\hline & Shortness of breath & 2231 & 2.19 \\
\hline & Chest pain & 1935 & 1.90 \\
\hline & Loss of smell, smell blindness & 1894 & 1.86 \\
\hline & Persistent fever (for 1 week or more) & 1663 & 1.63 \\
\hline & Loss of appetite/weight & 1333 & 1.31 \\
\hline & Loss of taste & 1689 & 1.66 \\
\hline \multirow[t]{4}{*}{ No. of symptoms* } & 1 & 11899 & 40.03 \\
\hline & 2 & 7062 & 23.76 \\
\hline & 3 & 4365 & 14.68 \\
\hline & 4 & 2481 & 8.34 \\
\hline \multirow[t]{4}{*}{ How did they feel when answering the questionnaire } & Well & 37599 & 66.50 \\
\hline & Normal & 12726 & 22.50 \\
\hline & Not at $100 \%$ & 6010 & 10.60 \\
\hline & Bad & 235 & 0.42 \\
\hline \multirow{7}{*}{$\begin{array}{l}\text { Use of healthcare resources put in place in the } \\
\text { context of the COVID-19 pandemic }\end{array}$} & None & 38955 & 64.25 \\
\hline & Have used an app set up for management of COVID-19 cases & 13044 & 21.51 \\
\hline & $\begin{array}{l}\text { Have called a telephone number set up for the management of COVID-19 } \\
\text { cases }\end{array}$ & 3399 & 5.60 \\
\hline & Have been to a public healthcare centre (including General Practitioners (GP)) & 2286 & 3.77 \\
\hline & Have been tested & 1108 & 1.82 \\
\hline & Have been to private doctor/healthcare centre & 973 & 1.60 \\
\hline & Have gone to the emergency room & 863 & 1.42 \\
\hline \multirow[t]{2}{*}{ For those tested, result of the test } & Negative & 621 & 57.76 \\
\hline & Positive & 454 & 42.23 \\
\hline
\end{tabular}

Number of cases (number of responses received per answer category) and percentage of the total responses obtained for each question. Please note that some of the questions were multiple choice.

*For the number of symptoms only answers up to four are presented, even if the percentage given was calculated for all the responses obtained. 
Table 3 Impact of the pandemic on the general population (continuation)

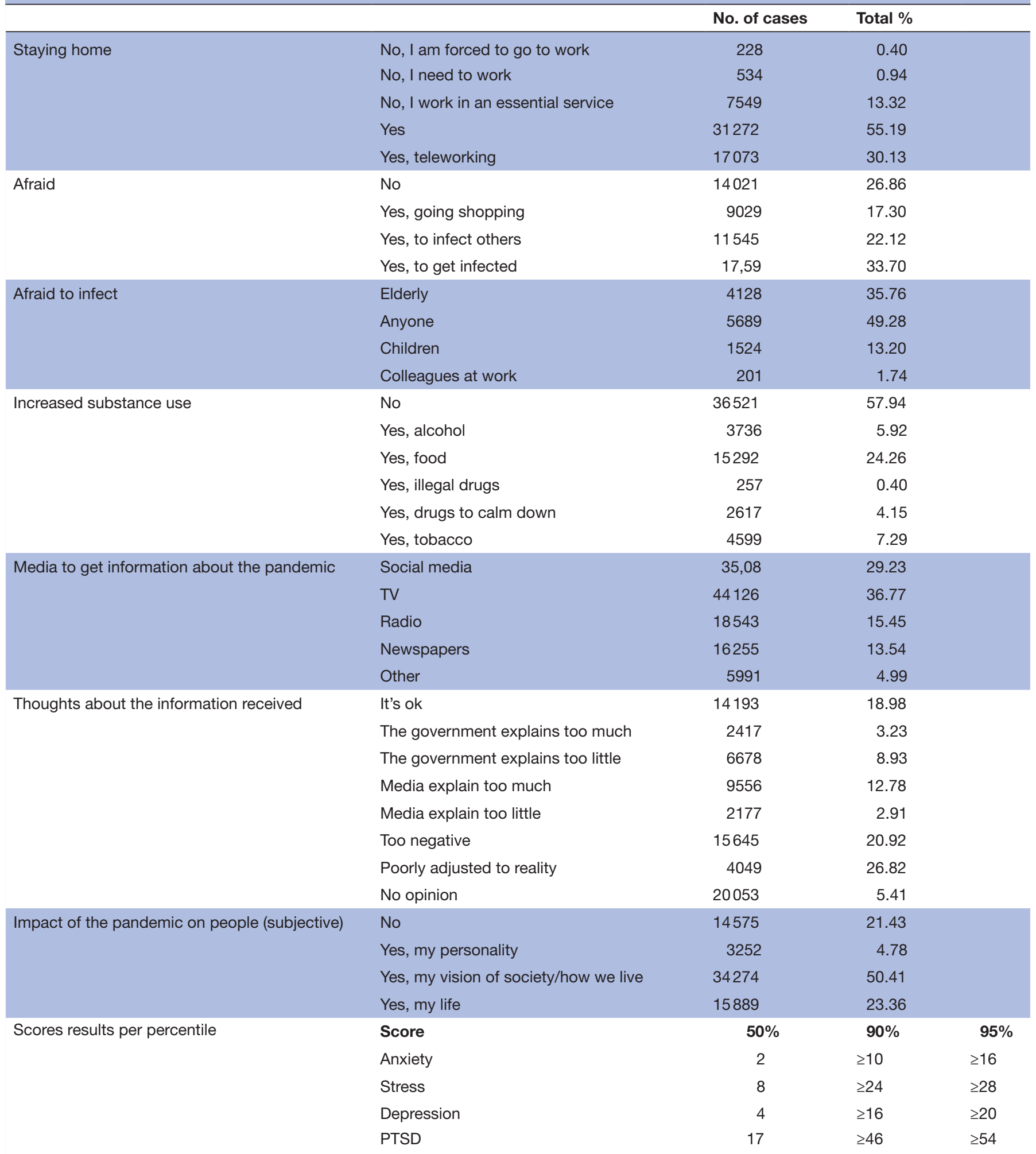

Number of cases (number of responses received per answer category) and percentage of the total responses obtained for each question. Please note that some of the questions were multiple choice.

"Score" refers to the data in the cells below, as there were several scores analyzed (there is one score for anxiety, one for stress, etc.). The $50 \%-90 \%$ and $95 \%$ in bold stand for the percentile, and the cells below are also referred to these. Example: The percentile $50 \%$ of the population sample had a value $=2$ of the score of Anxiety, only the $10 \%$ of the population sample had more than 10 (percentile 90), and etc.

PTSD, post-traumatic stress disorder. 


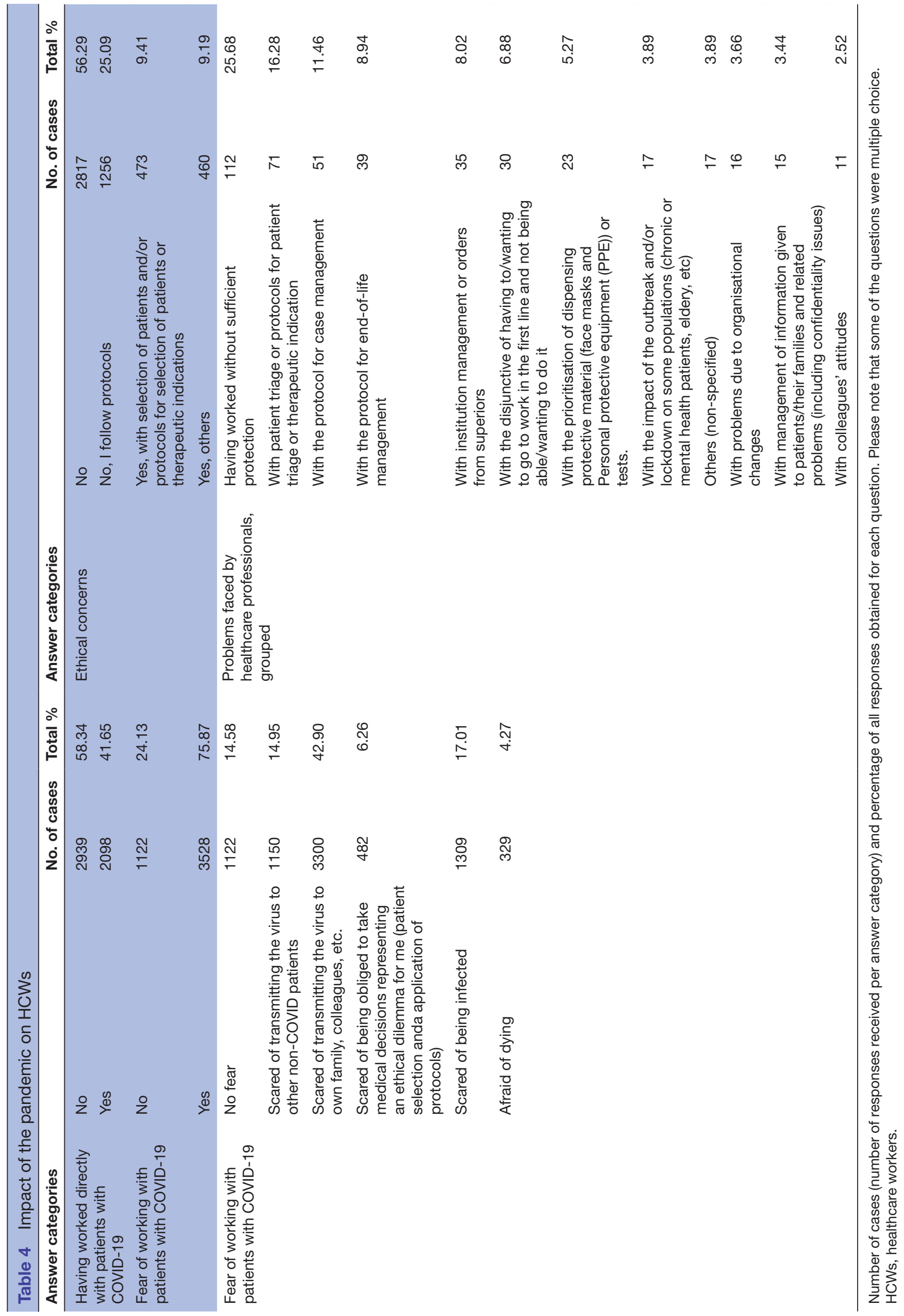


Table 5 Conditions statistically associated with the mental health score results

\begin{tabular}{|c|c|c|c|c|c|c|c|}
\hline \multirow[b]{2}{*}{ Factors: } & \multicolumn{7}{|c|}{ Statistically associated with: } \\
\hline & $\begin{array}{l}\text { Depression } \\
\text { index }\end{array}$ & $\begin{array}{l}\text { Anxiety } \\
\text { index }\end{array}$ & Stress index & $\begin{array}{l}\text { PTSD } \\
\text { index }\end{array}$ & $\begin{array}{l}\text { Evitation } \\
\text { index }\end{array}$ & $\begin{array}{l}\text { Intrusion } \\
\text { index }\end{array}$ & $\begin{array}{l}\text { Hyperarousal } \\
\text { index }\end{array}$ \\
\hline Risk & $P$ value & $P$ value & $P$ value & $P$ value & $P$ value & $P$ value & $P$ value \\
\hline Women & 0.019 & 0.003 & & 0.000 & 0.007 & 0.034 & 0.027 \\
\hline$<42$ years & & 0.008 & & & & & \\
\hline Caregivers & & 0.002 & 0.039 & 0.006 & & 0.050 & \\
\hline $\begin{array}{l}\text { Living in a middle to high density population } \\
\text { town }\end{array}$ & & 0.031 & & & & & \\
\hline Living in a shared apartment/house & & 0.006 & & & & & \\
\hline Living in a rented room & & 0.039 & & & & & \\
\hline $\begin{array}{l}\text { Being a healthcare worker and being afraid of } \\
\text { attending to patients with COVID- } 19\end{array}$ & 0.017 & & & & 0.023 & & \\
\hline $\begin{array}{l}\text { Having been in contact with a patient with } \\
\text { COVID-19 }\end{array}$ & & 0.006 & & 0.038 & & & \\
\hline $\begin{array}{l}\text { Having had symptoms compatible with } \\
\text { COVID-19 }\end{array}$ & 0.021 & 0.002 & & 0.008 & & & \\
\hline $\begin{array}{l}\text { Having used all healthcare resources put } \\
\text { in place in the context of the COVID-19 } \\
\text { pandemic }\end{array}$ & & & 0.039 & 0.008 & 0.007 & & 0.011 \\
\hline $\begin{array}{l}\text { Afraid (of getting infected, infecting others } \\
\text { and going shopping) }\end{array}$ & & 0.000 & 0.036 & 0.000 & 0.003 & 0.012 & 0.006 \\
\hline Being married & & 0.007 & & & & & \\
\hline Being a widow & & & & 0.020 & 0.011 & & \\
\hline Having a qualified job & & 0.008 & & & & & \\
\hline Having a PhD & 0.019 & 0.010 & & & 0.031 & & \\
\hline Feeling well & & 0.045 & & 0.037 & & & \\
\hline
\end{tabular}

PTSD, post-traumatic stress disorder.

More than 6\% of HCWs $(6.27 \%)$ were worried about taking medical decisions that represented an ethical problem for them, and nearly $18.60 \%$ of them declared that they had encountered ethical problems/dilemmas/issues while working. Of these, the younger the respondents, the higher the percentage, especially as regards patient triage and obligatory protocols $(\mathrm{p}<0.01)$. A total of 437 out of 5104 HCWs chose to explain the ethical problems and other issues they had experienced, as shown in table 4.

\section{Impact of the pandemic on mental health status}

Table 5 summarises the conditions found to be statistically significantly associated $(\mathrm{p}<0.05)$ with the mental health symptoms evaluated. On the basis of this table, we have identified seven target groups susceptible to benefitting from an intervention and which should be taken into account when designing new contention measures to cope with the pandemic: (1) women; (2) people aged under 42 years; (3) caregivers; (4) people working in essential services or non-qualified jobs; (5) people with a higher precariousness index; (6) COVID-19 patients; and (7) HCWs, especially those working with patients with COVID-19.

\section{DISCUSSION}

The current study aimed to identify the impacts of the COVID-19 pandemic on a wide range of health status 
dimensions in Catalonia while lockdown was in force. It is one of the most extensive surveys ever published, with a total of 56656 questionnaires analysed, but nevertheless has limitations that must be considered when interpreting the data. Thus, although our survey provides information about how people of different age ranges, and specifically woman and HCWs, have faced the pandemic in several spheres, it was not designed to be representative of a specific population. The survey was long, which may have generated fatigue and a high drop-out rate, although this also allowed us to collect a large volume of data. In addition, it was shared via social media, thus the sample of the population studied could not be controlled. However, although not ensuring representability, the snowball method proved to be a successful strategy that allowed us to rapidly reach a large number of people without exposing interviewers to infection. Another limitation is that the criteria used to establish ranges for some of the variables were statistical, in order to obtain balanced groups in terms of number of responses. This provides rigour but can be confusing because this segmentation is unusual and can lead to some degree of bias.

With regard to the impact on the socioeconomic sphere, the highest level of precariousness, which according to our results seems to occur in people aged less than 42 years, is striking. Of particular concern is the fact that $25 \%$ of respondents had experienced a decreased workload due to the epidemic situation, especially men, more of whom had lost more jobs or previously contracted assignments, and those aged less than 52 years, many of whom had been made redundant or put on temporary furlough. In addition, a quarter of respondents had no savings to protect them against contingencies, and up to $8.78 \%$ stated that they had applied for social benefits or that they would do so soon. Socioeconomic precariousness was found to be one of the factors associated with higher scores on the mental health indices, which is rather worrying given that the incidence of the pandemic was also more pronounced in the poorest neighbourhoods, at least in Barcelona. ${ }^{15}$

A value of approximately $20 \%$ for the population affected at mental health level seems consistent according to literature, ${ }^{71617}$ even if higher percentages have been found in some cases. ${ }^{18} 19$ Although no validated scales were used, the inclusion of 41 questions related to depression, anxiety, stress and PTSD symptoms allowed us to explore the impact on the mental health dimension. We identified up to seven target groups at higher risk of impaired mental health status and susceptible of benefitting from an intervention. A worse symptoms score was associated with the presence of symptoms compatible with COVID-19 or having used all the healthcare resources put in place. However, as a real intervention based on these assumptions would be very costly and logistically difficult, confirmed patients with COVID-19 might instead be a better target group for an intervention.

Being female, young and having unstable work or income have been shown to be significant correlators of psychological negative impact. ${ }^{18-21}$ Women are especially vulnerable as they bear the heavier burden of childcare and care of the elderly, suffer gender-based violence and have more precarious jobs. ${ }^{22}$ Crises exacerbate gender inequalities, including gender-based violence, increased care burden, inadequate access to health services and others. ${ }^{23-25}$ Moreover, women account for the majority of HCWs around the world, and those younger or with a childcare burden suffered psychological distress. ${ }^{26}{ }^{27} \mathrm{In}$ our setting, it was mostly women who were responsible for caring for others, and caregiver adults with a higher perception of the difficulty of quarantine for children and the whole family suffered more psychological distress than the other groups. Individual perception has previously been associated with stress levels and a negative behavioural and emotional impact on children, and it has been hypothesised that one of the causes could be the impact of the situation itself on both adults and their children (indirectly ${ }^{28}$ and directly ${ }^{29}$ ), along with the effects of school closures and the need to work from home with a lot of new inputs. Schools provide both education and counselling and promote and imply healthy habits that might not be continued at home. ${ }^{29}$

Given their frailty and increased risk of suffering COVID-19 if living in nursing homes or similar facilities, people aged more than 60 years represent the vast majority of all COVID-19 related deaths worldwide. ${ }^{30}$ The elderly are key in Mediterranean countries, such as ours, as they often take care of grandchildren when their parents go to work, so to quarantine and isolate them can be very disturbing for the whole of society. Moreover, COVID-19 and the consequences of isolating the elderly can be devastating for their mental health and as it contributes to a greater risk of morbidity, which may be even worse in the more disadvantaged populations. ${ }^{31} 32$ Although anxiety, depression and symptoms of avoidance coping have been reported for the elderly, ${ }^{33}{ }^{34}$ we found that younger people coped worse with the mental burden due to the COVID-19 pandemic, and the measures imposed to combat it, than older people. Older people have been shown to be more resilient than younger people in other outbreaks and major disasters, ${ }^{35}$ and our results also support this by showing that older people were less afraid of dying than younger ones. This could be due to the fact that the elderly have a greater sense of the meaning of life and that they tend to perceive time as being finite, which determines their priorities in terms of goals and behaviours. ${ }^{36}$ Young adults already face stressful life changes, and the pandemic has worsened this, even though one in five young adults might have been better off due to having been removed from external pressures, such as work and education and/or to having more time for close relationships. ${ }^{37}$ Several factors have been suggested to account for this worsening, including the perceived virus-related health risk ${ }^{37} 38$ and the decrease of physical and social activity due to lockdown and other restriction measures decreed by governments. ${ }^{38}{ }^{39} \mathrm{~A}$ study in France after 2 weeks of lockdown reported sleep 
problems and increased consumption of sleeping pills, with both being more frequent in people aged less than 35 years compared with older people. ${ }^{40}$ Similarly, Shanahan et al showed that a good group to be selected for intervention could be females, migrants and young adults with higher prepandemic emotional distress, including social exclusion. ${ }^{37}$

A non-negligible proportion of our respondents were HCWs who, in Europe, are mostly women. ${ }^{41}$ In addition to their obviously increased risk of becoming infected, ${ }^{42}$ being on the frontline against the SARS-CoV-2 pandemic may have put them under a great deal of pressure, thus increasing levels of anxiety and chronic stress (due to the overwork and suboptimal working conditions), which can last for to up to a year afterwards. ${ }^{114344}$

A study carried out in a cohort of $9138 \mathrm{HCWs}$ showed that $45.7 \%$ were at risk of suffering from a mental disorder, ${ }^{45}$ and another, which included 5450 HCWs, showed that $8.4 \%$ had experienced suicidal ideation and behaviour. ${ }^{46}$ In our study, being a HCW was found to be a positive factor for impaired mental health, especially for those working with patients with COVID-19 and afraid of infecting others, which has proved to have an impact on outcomes. ${ }^{47}$ This becomes worse as the tension in health systems increases, as frontline professionals work in a complex environment given the ethical challenges of COVID-19, eliciting different dimensions concerning ethical dilemmas related to the situation itself and the measures dictated by the government. ${ }^{48}$ The shortage of hospital beds was an important problem as it contributed to the case fatality rate and implied a triage of patients according to their likelihood of survival. ${ }^{49-51}$ The management of end-of-life situations was particularly worrying, as banning the support of relatives at the bedside had a very disturbing impact on patients and their families, but also on HCW mental health, workload, challenges and professional outcomes. ${ }^{52}$ According to our results, nearly 8 out of 10 HCWs declared that they were afraid of working with patients with COVID-19, especially given the risk of infecting others. Being obliged to work with lack of appropriate, or sufficient, personal protective equipment was one of the most frequent complaints of HCWs who shared their narratives on the ethical concerns they experienced. This low sense of security had previously been pointed out in small HCW cohorts elsewhere. ${ }^{53-55}$ We found differences between women and men in terms of the fear of transmitting the infection to others, and this could be related to women's jobs implying more exposure (as is the case for nurses, who in our cohort were mostly women). Those working in essential services also had higher psychological distress and this could be for the same reason, namely the low sense of security plus the fear of being at higher risk of contracting the disease.

Around $6.27 \%$ of respondents declared that their fear was of making medical decisions that represented an ethical problem for them, with this percentage being higher in younger people. One in five of our HCWs declared that they had experienced ethical problems, a value that is in line with other studies, ${ }^{52}{ }^{56}$ with approximately half of these being related to patient selection or patient triage protocols/therapeutic indications. In our opinion, this fact should also be explored more thoroughly and actively followed up to prevent health professionals from being put into similar situations in the future.

Our findings could be used to design and implement interventions to increase the resilience of the groups identified herein, as well as to prepare an appropriate organisational response. In this sense, some authors have published specific strategies that could be used to alleviate this suffering. ${ }^{52}{ }^{57-62}$ Some of the strategies at an individual and organisational level that could be actively implemented in the vulnerable populations identified are:

1. To identify individuals who may be more vulnerable to mental health difficulties or are part of the populations identified as being more vulnerable within each group/team/staff members and to provide them with appropriate care.

2. To provide education on mental hygiene, self-reflection and emotion-focused therapy using different tools (storytelling, music, meditation, etc).

3. To train in building resilience and foster a culture of resilience.

4. To promote mental health services and make them accessible to all. To plan a structured schedule to communicate existing resilience measures and support the programmes available and how to access them.

5. To draft and implement a systematic communication plan in order to provide timely, accurate, regular and evidence-based information on the situation and the response planned (including all scenarios). To perform training and inform about the tools available to ensure its implementation if they are involved in this response. This can be applied at all levels, including companies, health departments and hospitals, public health systems and at local and national government level.

6. To provide people with structured opportunities to debrief and talk after critical events, to hear about their real-time concerns and to engage them in collaborative approaches to decision making and problem solving.

\section{Conclusion}

We identified seven populations as being vulnerable and therefore likely to benefit from an intervention in the face of potential future outbreaks or other major disasters. Our study should open the door to the design of coping measures and the elaboration of strategy proposals with the full participation of those institutional leaders who are in a position to adapt policy to the real needs of the people at organisational, governmental and public health service levels.

Author affiliations

${ }^{1}$ Anaxomics Biotech SL, Barcelona, Catalonia, Spain 
${ }^{2}$ Experimental Tuberculosis Unit, UAB, Fundació Institut d'Investigació en Ciències de la Salut Germans Trias i Pujol, Badalona, Catalonia, Spain

${ }^{3}$ CIBER de Enfermedades Respiratorias (CIBERES), Centro de Investigación

Biomédica en Red, Madrid, Comunidad de Madrid, Spain

${ }^{4}$ Innate Immunity Group, Fundació Institut d'Investigació en Ciències de la Salut Germans Trias i Pujol, Badalona, Catalonia, Spain

${ }^{5}$ CIBER de Enfermedades Hepáticas y Digestivas (CIBEREHD), Centro de

Investigación Biomédica en Red, Madrid, Comunidad de Madrid, Spain

${ }^{6}$ Childhood Liver Oncology Group, Fundació Institut d'Investigació en Ciències de la

Salut Germans Trias i Pujol, Badalona, Catalonia, Spain

${ }^{7}$ Department of Microbiology, Hospital Universitari Germans Trias i Pujol, Badalona, Catalonia, Spain

${ }^{8}$ Fundació Lluita Contra la Sida, Badalona, Catalonia, Spain

${ }^{9}$ Facultat de Psicologia i Ciències de l'Educació, Universitat Oberta de Catalunya,

Barcelona, Catalonia, Spain

${ }^{10}$ Infectious Diseases Department, Hospital Universitari Germans Trias i Pujol, Badalona, Catalonia, Spain

${ }^{11}$ Parc Sanitari Sant Joan de Deu, Sant Boi de Llobregat, Catalonia, Spain

${ }^{12}$ Institut de Recerca Sant Joan de Deu, Parc Sanitari Sant Joan de Deu, Sant Boi de Llobregat, Catalonia, Spain

${ }^{13}$ CIBER de Salud Mental (CIBERSAM), Centro de Investigación Biomédica en Red, Madrid, Comunidad de Madrid, Spain

${ }^{14}$ CIBER de Epidemiología y Salud Pública (CIBERESP), Centro de Investigación

Biomédica en Red, Madrid, Comunidad de Madrid, Spain

\section{Twitter Cristina Vilaplana @tbexperimental}

Acknowledgements We would like to thank all the people who kindly shared the survey questionnaire via social media and/or completed it. We would also like to thank Jérôme Nigou, Valeria Bergalli, Paolo Salieri, Chiara Bertoldini and Harvey Evans, who kindly volunteered to translate/edit the questionnaire and website information into French, Italian and English, and to Mr Andrew Frankland for reviewing and editing the English grammar of the text.

Contributors M-RS, CA, MV and CV made substantial contributions to the conception or design of the work. JF, JLR, JMM, LA, M-RS, CA and CV made substantial contributions to the acquisition and analysis of data. M-RS, CA, P-JC, JAM-M, MV, BA, JU and AS-B made substantial contributions to the interpretation of data. MRS, CA and CV drafted the manuscript, and all the others authors revised it critically for important intellectual content. All authors gave final approval of the version to be published. $\mathrm{CV}$ is responsible for the overall content as guarantor.

Funding This work was supported by the Spanish Government-FEDER Funds through CV contract (CPII18/00031) and funding from the European Union's Horizon 2020 research and innovation programme under grant agreement no. 847762 through LA's contract.

Competing interests The salaries of JF, JLR and JAM-M are partially paid by the European Union's Horizon 2020 research and innovation programme under grant agreement no. 847 762. LA received support from the European Union's Horizon 2020 research and innovation programme under grant agreement no. 847 762 through her contract. JAM-M has a postdoctoral research contract from the Fundació Lluita contra la SIDA and has received honoraria for research/educational presentations by GILEAD Sciences and MSD. MV is the president of the Suicidal Conduct Committee of PSSJD. AS-B has received support from the Diputació de Barcelona via contracts or grants to carry out seven projects on mental health planning, from the Spanish Government-FEDER Funds through Instituto de Salud Carlos III (a grant to carry out a research project about mental health (PI19/00111 and PI15/00519)) and from the Catalan Government via an intensification research contract from the PERIS programme (SLT006/17/68), 2018-2020. He has acted as member of the Advisory Board of the Instituto de Salud Carlos III for the evaluation of research projects and as member of the Advisory Board of the Fundación Progreso y Salud for the evaluation of research projects. CV received support from the Spanish Government-FEDER Funds through CIBER Enfermedades Respiratorias and her contract (CPII18/00031), from the European Union's Horizon 2020 research and innovation programme for being the local PI of the Comix study (conducted within the EpiPose project (GA 101003688)) and has acted as an expert member of the COVID-19 crisis committee of the IGTP. MR-S, CA, P-JC, BA and JU declared no competing interests.

Patient consent for publication Not applicable.

Ethics approval PI-20-114, from the Germans Trias i Pujol Hospital Ethics Committee.
Provenance and peer review Not commissioned; externally peer reviewed.

Data availability statement Data are available upon reasonable request. Data are available in a public, open access repository. All data relevant to the study are included in the article or uploaded as supplementary information.

Supplemental material This content has been supplied by the author(s). It has not been vetted by BMJ Publishing Group Limited (BMJ) and may not have been peer-reviewed. Any opinions or recommendations discussed are solely those of the author(s) and are not endorsed by BMJ. BMJ disclaims all liability and responsibility arising from any reliance placed on the content. Where the content includes any translated material, BMJ does not warrant the accuracy and reliability of the translations (including but not limited to local regulations, clinical guidelines, terminology, drug names and drug dosages), and is not responsible for any error and/or omissions arising from translation and adaptation or otherwise.

Open access This is an open access article distributed in accordance with the Creative Commons Attribution Non Commercial (CC BY-NC 4.0) license, which permits others to distribute, remix, adapt, build upon this work non-commercially, and license their derivative works on different terms, provided the original work is properly cited, appropriate credit is given, any changes made indicated, and the use is non-commercial. See: http://creativecommons.org/licenses/by-nc/4.0/.

ORCID iDs

Judith Farrés http://orcid.org/0000-0002-0958-0510

Cristina Vilaplana http://orcid.org/0000-0002-2808-7270

\section{REFERENCES}

1 Ministerio de Sanidad Servicios Sociales E Igualdad; Ministerio de Economía Y Competitividad; Instituto de Salud Carlos III. Situación del COVID-19 en España. Available: https://covid19.isciii.es/

2 Health Department of the Catalan Government (Institution). Comunicat del Departament de Salut, 30 de març de 2020, 2020. Available: https://govern.cat/salapremsa/notes-premsa/383715/ comunicat-del-departament-salut

3 European Centre for Disease Prevention and Control. Novel coronavirus disease 2019 (COVID-19) pandemic : increased transmission in the EU / EEA and the UK - sixth update, 2020. Available: https://www.ecdc.europa.eu/sites/default/files/documents/ RRA-sixth-update-Outbreak-of-novel-coronavirus-disease-2019COVID-19.pdf

4 CDC. Patients with COVID-19. CDC website, 2020. Available: https:// www.cdc.gov/coronavirus/2019-ncov/hcp/disposition-hospitalizedpatients.html\%0D\%0A\%0D\%0A\%0D\%0A [Accessed 20 Mar 2020].

5 Van Bortel T, Basnayake A, Wurie F, et al. Psychosocial effects of an Ebola outbreak at individual, community and international levels. Bull World Health Organ 2016;94:210-4.

6 Rubin GJ, Wessely S. The psychological effects of quarantining a City. BMJ 2020;368:m313-2.

7 Wang C, Pan R, Wan X, et al. Immediate psychological responses and associated factors during the initial stage of the 2019 coronavirus disease (COVID-19) epidemic among the general population in China. Int J Environ Res Public Health 2020;17. doi:10.3390/ijerph17051729. [Epub ahead of print: 0603 2020].

8 Sim K, Huak Chan Y, Chong PN, et al. Psychosocial and coping responses within the community health care setting towards a national outbreak of an infectious disease. J Psychosom Res 2010;68:195-202.

9 Brooks SK, Webster RK, Smith LE, et al. The psychological impact of quarantine and how to reduce it: rapid review of the evidence. Lancet 2020;395:912-20.

10 Leung GM, Lam T-H, Ho L-M, et al. The impact of community psychological responses on outbreak control for severe acute respiratory syndrome in Hong Kong. J Epidemiol Community Health 2003;57:857-63.

11 McAlonan GM, Lee AM, Cheung V, et al. Immediate and sustained psychological impact of an emerging infectious disease outbreak on health care workers. Can J Psychiatry 2007;52:241-7.

12 Gibbons JD, Chakraboti S. Nonparametric statistical inference. 5 edn. Boca Raton: Chapman \& Hall/CRC Press, Taylor \& Francis Group, 2011.

13 Hollander M, Wolfe D. Nonparametric statistical methods. 2 edn, 1999.

14 INEbase / demography and population /Population figures and demographic Censuses /Population figures / latest data.

15 Marí-Dell'olmo M, Gotsens M, Pasarín MI. Socioeconomic inequalities in COVID-19 in a European urban area: two waves, two patterns. Int J Environ Res Public Health 2021. 
16 González-Sanguino C, Ausín B, Castellanos Miguel Ángel, et al. Mental health consequences during the initial stage of the 2020 coronavirus pandemic (COVID-19) in Spain. Brain Behav Immun 2020;87:172-6.

17 Wang C, Pan R, Wan X. A longitudinal study on the mental health of general population during the COVID-19 epidemic in China. Brain Behav Immun 2020.

18 Rodríguez-Rey R, Garrido-Hernansaiz H, Collado S. Psychological impact and associated factors during the initial stage of the coronavirus (COVID-19) pandemic among the general population in Spain. Front Psychol 2020;11:1540

19 Jacques-Aviñó C, López-Jiménez T, Medina-Perucha L, et al. Gender-based approach on the social impact and mental health in Spain during COVID-19 lockdown: a cross-sectional study. BMJ Open 2020;10:e044617.

20 Ramírez LPG, Arriaga RJM, Hernández-Gonzalez MA. Psychological distress and signs of post-traumatic stress in response to the COVID-19 health emergency in a Mexican sample. Psychol Res Behav Manag 2020.

21 Cao W, Fang Z, Hou G. The psychological impact of the COVID-19 epidemic on college students in China. Psychiatry Res 2020.

22 Walter LA, McGregor AJ. Sex- and gender-specific observations and implications for COVID-19. West J Emerg Med 2020;21:507-9.

23 Peterman A, Potts A, Donnell MO. Working paper 528 April 2020 pandemics and violence against women and children. Cent Glob Dev 2020:43.

24 Davies SE, Bennett B. A gendered human rights analysis of Ebola and Zika: locating gender in global health emergencies. Int Aff2016.

25 Mollona E, Aivazidou E, Barberio V. A policy framework for tackling the economic and social impact of the COVID-19 crisis 2019.

26 Kisely S, Warren N, McMahon L, et al. Occurrence, prevention, and management of the psychological effects of emerging virus outbreaks on healthcare workers: rapid review and meta-analysis. BMJ 2020;369:m1642.

27 Davico C, Ghiggia A, Marcotulli D, et al. Psychological impact of the COVID-19 pandemic on adults and their children in Italy. Front Psychiatry 2021;12:1-8.

28 Spinelli M, Lionetti F, Pastore M, et al. Parents' stress and children's psychological problems in families facing the COVID-19 outbreak in Italy. Front Psychol 2020;11:1713.

29 Wang G, Zhang Y, Zhao J, et al. Mitigate the effects of home confinement on children during the COVID-19 outbreak. Lancet 2020;395:945-7

30 Kang SJ, Jung SI. Age-Related morbidity and mortality among patients with COVID-19. Infect Chemother 2020;52:154.

31 Armitage R, Nellums LB. COVID-19 and the consequences of isolating the elderly. Lancet Public Health 2020;5:e256.

32 Petretto DR, Pili R, Ageing PR. Ageing and COVID-19: what is the role for elderly people? Geriatrics 2020;5:25.

33 Meng H, Xu Y, Dai J. Analyze the psychological impact of COVID-19 among the elderly population in China and make corresponding suggestions. Psychiatry Res 2020.

34 Bobes-Bascarán T, Sáiz PA, Velasco A. Early psychological correlates associated with COVID-19 in a Spanish older adult sample. Am J Geriatr Psychiatry 2020:1-12.

35 Cohen O, Geva D, Lahad M, et al. Community Resilience throughout the Lifespan--The Potential Contribution of Healthy Elders. PLoS One 2016;11:e0148125-14.

36 Fernández-Aguilar L, Ricarte J, Ros L, et al. Emotional differences in young and older adults: films as mood induction procedure. Front Psychol 2018;9:1110.

37 Shanahan L, Steinhoff A, Bechtiger L, et al. Emotional distress in young adults during the COVID-19 pandemic: evidence of risk and resilience from a longitudinal cohort study. Psychol Med 2020:1-10.

38 Commodari E, La Rosa VL. Adolescents in quarantine during COVID-19 pandemic in Italy: perceived health risk, beliefs, psychological experiences and expectations for the future. Front Psychol 2020;11:2480.

39 Maugeri G, Castrogiovanni P, Battaglia G, et al. The impact of physical activity on psychological health during Covid-19 pandemic in Italy. Heliyon 2020;6:e04315.

40 Beck F, Léger D, Fressard L, et al. Covid-19 health crisis and lockdown associated with high level of sleep complaints and hypnotic uptake at the population level. J Sleep Res 2021;30:e13119.
41 Rossi R, Socci V, Pacitti F, et al. Mental health outcomes among frontline and second-line health care workers during the coronavirus disease 2019 (COVID-19) pandemic in Italy. JAMA Netw Open 2020;3:e2010185.

42 Xiao J, Fang M, Chen Q, et al. Sars, MERS and COVID-19 among healthcare workers: a narrative review. J Infect Public Health 2020;13:843-8.

43 Simione L, Gnagnarella C. Differences between health workers and general population in risk perception, behaviors, and psychological distress related to COVID-19 spread in Italy. Front Psychol 2020;11:2166.

44 Pappa S, Ntella V, Giannakas T. Prevalence of depression, anxiety, and insomnia among healthcare workers during the COVID-19 pandemic: a systematic review and meta-analysis. Brain Behav Immun 2020.

45 Alonso J, Vilagut G, Mortier P. Mental health impact of the first wave of COVID-19 pandemic on Spanish healthcare workers: a large cross-sectional survey. Rev Psiquiatr Salud Ment 2021.

46 Mortier P, Vilagut G, Ferrer M, et al. Thirty-day suicidal thoughts and behaviors among hospital workers during the first wave of the Spain COVID-19 outbreak. Depress Anxiety 2021;38:528-44.

47 Ramaci T, Barattucci M, Ledda C. Social stigma during COVID-19 and its impact on HCWs outcomes. Sustain 2020.

48 Robert R, Kentish-Barnes N, Boyer A, et al. Ethical dilemmas due to the Covid-19 pandemic. Ann Intensive Care 2020;10.

49 Zanin M, Xiao C, Liang T. The public health response to the COVID-19 outbreak in mainland China: a narrative review. $J$ Thorac Dis 2020.

50 Verweij M, van de Vathorst S, Schermer M, et al. Ethical advice for an intensive care triage protocol in the COVID-19 pandemic: lessons learned from the Netherlands. Public Health Ethics 2020;13:157-65

51 Vincent J-L, Creteur J. Ethical aspects of the COVID-19 crisis: how to deal with an overwhelming shortage of acute beds. Eur Heart $J$ 2020;9:248-52.

52 Owens IT. Supporting nurses' mental health during the pandemic. Nursing 2020;50:54-7.

53 JÁ M-L, Lázaro-Pérez C, Gómez-Galán J. Psychological impact of COVID-19 emergency on health professionals: Burnout incidence at the most critical period in Spain. J Clin Med 2020;9

54 Nowicki GJ, Ślusarska B, Tucholska K, et al. The severity of traumatic stress associated with covid-19 pandemic, perception of support, sense of security, and sense of meaning in life among nurses: research protocol and preliminary results from Poland. Int J Environ Res Public Health 2020;17. doi:10.3390/ijerph17186491. [Epub ahead of print: 0709 2020].

55 Delgado D, Wyss Quintana F, Perez G, et al. Personal safety during the covid-19 pandemic: realities and perspectives of healthcare workers in Latin America. Int J Environ Res Public Health 2020;17. doi:10.3390/ijerph17082798. [Epub ahead of print: 1804 2020].

56 Epstein EG, Delgado S. Understanding and addressing moral distress. Online J Issues Nurs2010.

57 Tomlin J, Dalgleish-Warburton B, Lamph G. Psychosocial support fo healthcare workers during the COVID-19 pandemic. Front Psychol 2020;11:1960.

58 Maves RC, Downar J, Dichter JR, et al. Triage of scarce critical care resources in COVID-19 an implementation guide for regional allocation: an expert panel report of the task force for mass critical care and the American College of chest physicians. Chest 2020;158:212-25.

59 Johns Hopkins Berman Institute of Bioethics. Resources for frontline clinicians, 2020.

60 Chmitorz A, Kunzler A, Helmreich I, et al. Intervention studies to foster resilience - A systematic review and proposal for a resilience framework in future intervention studies. Clin Psychol Rev 2018:59:78-100.

61 Norris FH, Stevens SP, Pfefferbaum B, et al. Community resilience as a metaphor, theory, set of capacities, and strategy for disaster readiness. Am J Community Psychol 2008;41:127-50.

62 Ye J. Pediatric mental and behavioral health in the period of quarantine and social distancing with COVID-19. JMIR Pediatr Parent 2020;3:e19867. 